\title{
INTERSECTION HOMOLOGY BETTI NUMBERS
}

\author{
ALAN H. DURFEE \\ (Communicated by Eric Friedlander)
}

\begin{abstract}
A generalization of the formula of Fine and Rao for the ranks of the intersection homology groups of a complex algebraic variety is given. The proof uses geometric properties of intersection homology and mixed Hodge theory.
\end{abstract}

The middle-perversity intersection homology with integral coefficients of a compact complex $n$-dimensional algebraic variety $X$ with isolated singularities is well known to be [GM1]

$$
I H_{k}(X) \cong \begin{cases}H_{k}(X) & \text { for } k>n, \\ \operatorname{Im}\left\{H_{n}(U) \rightarrow H_{n}(X)\right\} & \text { for } k=n, \\ H_{k}(U) & \text { for } k<n\end{cases}
$$

where $U$ is the complement of the singular set in $X$. Fine and Rao [FR] find a formula for the ranks of the intersection homology groups of $X$ in terms of the ordinary Betti numbers of a resolution of singularities of $X$. The formula is an application of (1), which is a purely geometric fact, and mixed Hodge theory.

This paper contains a mild generalization of their formula to the case when the variety has nonisolated singularities. First, (1) is generalized to nonisolated singularities (Proposition 3). The proof uses a basic geometric property of intersection homology (Lemma 1) and a standard exact sequence of mixed Hodge theory (Lemma 2). A generalization of the Fine-Rao formula for the Betti numbers (Proposition 4) follows easily. The proof of the formula given in this paper makes clear the separate roles played by geometry and mixed Hodge theory.

Let $X$ be a complex algebraic variety which is not necessarily compact (i.e., a reduced separable scheme of finite type over the complex numbers), and suppose that the complex dimension of $X$ is $n$. Let $Z$ be a closed subvariety of dimension $\leq d$, and let $U=X \backslash Z$. We let $I H_{c}^{k}(X)=I H_{2 n-k}(X)$ (respectively, $\left.I H^{k}(X)=I H_{2 n-k}^{\mathrm{BM}}(X)\right)$, where $I H_{k}(X)$ (respectively, $I H_{k}^{\mathrm{BM}}(X)$ ) denotes the middle-perversity intersection homology group of $X$ (respectively, middle-perversity Borel-Moore intersection homology group), and similarly for open subsets of $X$. For compact $X$ with isolated singularities, formula (1)

Received by the editors June 3, 1993.

1991 Mathematics Subject Classification. Primary 14E32.

The author was partially supported by NSF Grant DMS-8901903.

(C) 1995 American Mathematical Society $0002-9939 / 95 \$ 1.00+\$ .25$ per page 
thus becomes

$$
I H^{k}(X) \cong \begin{cases}H^{k}(X) & \text { for } k>n \\ \operatorname{Im}\left\{H^{n}(X) \rightarrow H^{n}(U)\right\} & \text { for } k=n \\ H^{k}(U) & \text { for } k<n\end{cases}
$$

Let $I C_{X}^{\text {top }}$ be the sheaf complex defined by $\left(I C_{X}^{\text {top }}\right)^{k}(V)=I C_{-k}^{\mathrm{BM}}(V)$ for $V \subset X$ open so that $I H^{k}(X)$ is the hypercohomology group $H^{k}\left(X, I C_{X}^{\text {top }}\right)$, etc. Let $i: Z \hookrightarrow X$ and $j: U \hookrightarrow X$. We abbreviate $R i_{*}$ by writing $i_{*}$ and so forth, and we let $I C_{X}=I C_{X}^{\text {top }}[-n]$ as in [BBD].

The following lemma expresses a basic geometric property of intersection homology. If $Z$ has a mapping cylinder neighborhood $N$ in $X$ (see [DS]), then a geometric argument similar to [GM2,1.7] shows the equivalent result $I H_{k}^{\mathrm{BM}}(N)=0$ for $k \leq n-d$ and $I H_{k}(N)=0$ for $k \geq n+d$.

Lemma 1. If $X$ is an algebraic variety of dimension $n$ and $U$ an open subvariety with $Z=X \backslash U$ a closed subvariety of dimension $\leq d$, then (with integer coefficients)

$$
\begin{aligned}
I H_{c}^{k}(X) & \cong I H_{c}^{k}(U) \quad \text { for } k>n+d, \\
I H_{c}^{n+d}(X) & \leftarrow I H_{c}^{n+d}(U), \\
I H^{n-d}(X) & \hookrightarrow I H^{n-d}(U), \\
I H^{k}(X) & \cong I H^{k}(U) \text { for } k<n-d .
\end{aligned}
$$

Proof. There is an exact triangle [BBD, 1.4.3.4]

$$
j_{!} j^{*} I C_{X} \rightarrow I C_{X} \rightarrow i_{*} i^{*} I C_{X}
$$

Taking $H_{c}^{k-n}(X,-)$ gives

$$
\rightarrow H_{c}^{k-n}\left(X, j_{!} j^{*} I C_{X}\right) \rightarrow H_{c}^{k-n}\left(X, I C_{X}\right) \rightarrow H_{c}^{k-n}\left(X, i_{*} i^{*} I C_{X}\right) \rightarrow
$$

which gives

$$
\rightarrow I H_{c}^{k}(U) \rightarrow I H_{c}^{k}(X) \rightarrow H_{c}^{k-n}\left(Z, i^{*} I C_{X}\right) \rightarrow .
$$

Now $H^{k-n}\left(i^{*} I C_{X}\right) \mid Z=0$ as sheaf for $k-n \geq-d$ by [BBD, p. 9]. Since $Z$ has complex dimension $\leq d$, we have $H^{k-n}\left(Z, i^{*} I C_{X}\right)=0$ for $k-n \geq d$. This proves the first part of the lemma.

Similarly there is an exact triangle

$$
i_{*} i ! I C_{X} \rightarrow I C_{X} \rightarrow j_{*} j ! I C_{X}
$$

Taking $H^{k-n}(X,-)$ gives

$$
\rightarrow H^{k-n}\left(X, i_{*} i^{!} I C_{X}\right) \rightarrow H^{k-n}\left(X, I C_{X}\right) \rightarrow H^{k-n}\left(X, j_{*} j^{!} I C_{X}\right) \rightarrow
$$

which gives

$$
\rightarrow H^{k-n}\left(Z, i ! I C_{X}\right) \rightarrow I H^{k}(X) \rightarrow I H^{k}(U) \rightarrow .
$$

Now $H^{k-n}\left(i ! I C_{X}\right) \mid Z=0$ as sheaf for $k-n \leq-d$ by [BBD, p. 9], so $H^{k-n}\left(Z, i ! I C_{X}\right)=0$ for $k-n \leq-d$. This proves the last part of the lemma.

Next we need a basic property of the mixed Hodge structure on intersection cohomology. By work of Saito [Sai2], the intersection cohomology groups of 
an algebraic variety have a mixed Hodge structure. In particular, they have a weight filtration $\cdots \subset W_{m} \subset W_{m+1} \subset \cdots$. This filtration is functorial for algebraic maps, and $\mathrm{Gr}_{m}^{W}=W_{m} / W_{m-1}$ is an exact functor.

Lemma 2. If $X$ is a complex algebraic variety and $U$ an open subvariety, then for all $k$ (with rational coefficients):

(1) $\mathrm{Gr}_{k}^{W} I H_{c}^{k}(U) \hookrightarrow \mathrm{Gr}_{k}^{W} I H_{c}^{k}(X)$.

(2) $\operatorname{Gr}_{k}^{W} I H^{k}(X) \rightarrow \mathrm{Gr}_{k}^{W} I H^{k}(U)$.

The first part of this lemma is a generalization of the fact that for a smooth compactification $X$ of a smooth $U$, the classes in $H^{k}(U)$ of weight $k$ are restrictions of classes in $H^{k}(X)$ of weight $k$ [ Del, 3.2.17]. The proof of the lemma uses Saito's theory of mixed Hodge modules [Sai2], which is parallel to the theory of mixed perverse sheaves [BBD]. We recall that $I C_{X}$ as a mixed Hodge module has pure weight $n$, where $n$ is the dimension of $X$. Also if $f$ is a map of algebraic varieties, then $f_{!}$and $f^{*}$ preserve weight $\leq m$ and $f_{*}$ and $f^{!}$preserve weight $\geq m$. Finally, if $K$ is a complex of weight $\leq m$ (respectively, $\geq m$ ), then $H^{k}(K)$ has weight $\leq m+k$ (respectively, $\geq m+k$ ).

Proof. Let $a: X \rightarrow p t$. The exact triangle (2) is an exact triangle of mixed Hodge modules [Sai1,4.4.1]. Taking $a_{!}$and $H^{k-n}(-)$ is the same as taking $H_{c}^{k-n}(X,-)$, so all terms of the exact sequence (3) have weight $\leq k$, and taking $\mathrm{Gr}_{k}^{W}$ proves the first part of the lemma.

Similarly taking $a_{*}$ and $H^{k-n}(-)$ of the exact triangle (4) is the same as taking $H^{k}(X,-)$, so all terms of exact sequence (5) have weight $\geq k$, and taking $\mathrm{Gr}_{k}^{W}$ proves the second part of the lemma.

Combining Lemmas 1 and 2 yields the following proposition.

Proposition 3. If $X$ is a compact algebraic variety of dimension $n$ and $U$ an open smooth subvariety with $Z=X \backslash U$ of dimension $\leq d$, then (with rational coefficients)

$$
I H^{k}(X) \cong \begin{cases}H_{c}^{k}(U) & \text { for } k>n+d, \\ \operatorname{Gr}_{n+d}^{W} H_{c}^{n+d}(U) & \text { for } k=n+d, \\ \operatorname{Gr}_{n-d}^{W} H^{n-d}(U) & \text { for } k=n-d, \\ H^{k}(U) & \text { for } k<n-d .\end{cases}
$$

The proposition follows since $I H^{k}(X)$ has pure weight $k$. Note that $H_{c}^{k}(U)$ can be replaced by $H^{k}(X, Z) \cong H^{k}(X)$ since $k \geq n+d$. The proposition depends on mixed Hodge theory and hence is valid only over the rational numbers. Is there an integral version of this proposition, with a geometric proof similar to Formula 1 ?

The weighted Euler characteristic of a space $Y$ with mixed Hodge structure is defined by

$$
\mathcal{X}_{m}(Y)=\sum_{i}(-1)^{i} \operatorname{dim} \mathrm{Gr}_{m}^{W} H^{i}(Y)
$$

where $W$ is the weight filtration. Similarly we define $\mathcal{X}_{m}^{c}(Y)$ using $H_{c}^{i}(Y)$ in place of $H^{i}(Y)$. The following proposition gives a formula for the ranks of the outer intersection homology groups of an algebraic variety in terms of the ordinary Betti numbers of a resolution of its singularities. 
Proposition 4. Let $X$ be a compact variety of dimension $n$, and let $Z$ be a closed subvariety of dimension $\leq d$ containing the singular set of $X$. Let $\pi: \widetilde{X} \rightarrow X$ be an algebraic map with $\tilde{X}$ smooth and $\pi^{-1}(Z)=E=E_{1} \cup \cdots \cup E_{r}$ a divisor with normal crossings. Suppose that $\pi$ is an analytic isomorphism of $\widetilde{U}=\widetilde{X} \backslash E$ to $U=X \backslash Z$. Then for $m \geq n+d$

$$
\operatorname{dim} I H^{m}(X)=\operatorname{dim} H^{m}(\tilde{X})-\sum_{i}(-1)^{i} \operatorname{dim} H^{m}\left(E_{i}\right) .
$$

Proof. By Proposition 3 and the fact that $I H^{k}(X)$ is pure and the weights on $H_{c}^{k}(U)$ are $\leq k$ for all $k$,

$$
(-1)^{m} \operatorname{dim} I H^{m}(X)=(-1)^{m} \operatorname{dim} \mathrm{Gr}_{m}^{W} I H_{c}^{m}(U)=\mathcal{X}_{m}^{c}(U)
$$

for $m \geq n+d$. The exact sequence of mixed Hodge structures

$$
\rightarrow H_{c}^{k}(\tilde{U}) \rightarrow H^{k}(\tilde{X}) \rightarrow H^{k}(E) \rightarrow
$$

gives

$$
\mathcal{X}_{m}^{c}(\tilde{U})=\mathcal{X}_{m}(\tilde{X})-\mathcal{X}_{m}(E) .
$$

Since $\widetilde{X}$ is compact and smooth, its cohomology is pure and

$$
\mathcal{X}_{m}(\tilde{X})=(-1)^{m} \operatorname{dim} H^{m}(\tilde{X}) .
$$

The exceptional set $\tilde{Z}$ has a simplicial resolution

$$
E_{\bullet}=\cdots \rightarrow E_{[l]} \rightarrow E_{[l-1]} \rightarrow \cdots
$$

where

$$
E_{[l]}=\coprod_{|I|=I+1}\left(\bigcap_{i \in I} E_{i}\right)
$$

Thus,

$$
\mathcal{X}_{m}(\tilde{Z})=\sum(-1)^{i} \mathcal{X}_{m}\left(E_{i}\right)=\sum(-1)^{i}(-1)^{m} \operatorname{dim} H^{m}\left(E_{i}\right)
$$

which finishes the proof.

By duality this gives the ranks of the groups in dimensions $\leq n-d$. The same argument also gives a formula for the Hodge numbers.

What about the missing middle-dimensional Betti numbers? The following is a rather naive estimate of the rank of intersection homology groups.

Proposition 5. Let $X$ be a compact algebraic variety, let $U \subset X$ be a smooth open subset, and let $\tilde{X} \rightarrow X$ be a resolution of singularities. Then for all $k$,

$$
\max \left\{\operatorname{dim} \mathrm{Gr}_{k}^{W} H^{k}(U), \operatorname{dim} \mathrm{Gr}_{k}^{W} H_{c}^{k}(U)\right\} \leq \operatorname{dim} I H^{k}(X) \leq \operatorname{dim} H^{k}(\tilde{X}) .
$$

This follows from Lemma 2 and the fact that the decomposition theorem implies that the intersection homology of $X$ is a direct summand of the ordinary homology of $\tilde{X}$ [BBD]. The lower bound is realized when the computation of intersection homology is essentially reduced to (1) or Proposition 4. The upper bound is realized if the resolution $\tilde{X} \rightarrow X$ is small. For example, Schubert varieties have small resolutions [BFL]. If the normalization of a variety is smooth, then it is a small resolution as well. 


\section{REFERENCES}

[BBD] A. A. Beilinson, J. Bernstein, and P. Deligne, Faisceaux pervers, Astérisque 100 (1982).

[BFL] P. Bressler, M. Finkelberg, and V. Lunts, Vanishing cycles in grassmannians, Duke Math. J. 161 (1990), 763-777.

[Del] P. Deligne, Théorie de Hodge. II, Inst. Hautes Études Sci. Publ. Math. 40 (1972), 5-57.

[DS] A. H. Durfee and M. Saito, Mixed Hodge structures on the intersection cohomology of links, Compositio Math. 76 (1990), 49-67.

[FR] J. Fine and P. Rao, On intersection homology at isolated singularities, Algebras, Groups and Geometries 5 (1988), 329-340.

[GM1] M. Goresky and R. MacPherson, Intersection homology theory, Topology 19 (1980), 135-162.

[GM2] __ Morse theory and intersection homology, Astérisque 101 (1983), 135-192.

[Sai1] M. Saito, Mixed Hodge modules, Publ. Res. Inst. Math. Sci. 26 (1990), 221-333.

[Sai2] _ Mixed Hodge modules and applications, Proc. Internat. Congr. Math. (Kyoto 1990) (I. Satake, ed.), Springer-Verlag, Tokyo, 1991, pp. 725-734.

Department of Mathematics, Mount Holyoke College, South Hadley, Massachusetts 01075

E-mail address: adurfee@mtholyoke.edu 